\title{
Computational issues related to iterative coupling of subsurface and channel flows
}

\section{Ronald H.W. Hoppe ${ }^{1}$, Paulo Porta ${ }^{2}$, Yuri Vassilevski ${ }^{3}$}

1 Lehrstuhl für Angewandte Analysis mit Schwerpunkt Numerik, Universität Augsburg, 86159 Augsburg, Germany and

Department of Mathematics, University of Houston, Houston, TX 77204-3008, USA

e-mail: hoppe@math.uni-augsburg.de

2 Zentrum für Unweltsimulation, Universität Augsburg, 86159 Augsburg, Germany e-mail: porta@math.uni-augsburg.de

3 Institute of Numerical Mathematics, Russian Academy of Sciencies, 119991 Moscow, Russia and

Zentrum für Unweltsimulation, Universität Augsburg, 86159 Augsburg, Germany e-mail: vasilevs@dodo.inm.ras.ru

Received: May 2006 / Revised version: September 2006 - (C) 2007 The Authors ${ }^{\star}$

Abstract. We consider solution techniques for the coupling of Darcy and Stokes flow problems. The study was motivated by the simulation of the interaction between channel flow and subsurface water flow for realistic data and arbitrary interfaces between the two different flow regimes. Here, the emphasis is on the efficient iterative solution of the coupled problem based on efficient solvers for the discrete Stokes and Darcy problems.

\section{Introduction}

Coupling different types of flows is an important issue in many applications. The most convenient way of dealing with coupled flow problems consists in the definition of two non-overlapping subdomains where different laws of flow are satisfied. The solutions in the subdomains must satisfy appropriate matching conditions at the interface. We consider the case of coupling Stokes and Darcy flows [3-6, 8,9,12,15]. In [9], Layton, Schieweck and Yotov provide a variational formulation for which weak solutions are shown to exist and which can be used as a base for a domain decomposition strategy

^ This is a "Springer Open Choice" article. Unrestricted non-commercial use, distribution, and reproduction in any medium is permitted, provided the original author and source are credited. 
for its approximate solution [13]. In [9], the proposed finite element method imposes the interface condition by using Lagrange multipliers which are used as boundary values for each subproblem. The subproblems are written in weak form and solved independently. The interface data are relaxed through an iterative process.

An alternative approach consists in a definition of the coupled problem where the solutions are matched directly without Lagrange multipliers. An appealing feature of this approach is that it suggests a natural way for the iterative solution based on the Neumann-Dirichlet iteration [3-6,12]. In physical terms, the solution procedure iteratively matches fluxes under the assumption of normal stress continuity. Numerical studies focused on coupling Darcy flow with shallow water equations [3,4], and on coupling Darcy and Stokes flows in 2D [5,6] as well as in 3D [12]. A thorough analysis of the associated interface problems can be found in [6]. From a practical standpoint, the advantage of the Neumann-Dirichlet iteration is that it allows us to use independent software realizing the subdomain solvers: they exchange minimal interface data. This is indispensable in industrial applications where commercial codes for Stokes and Darcy solves can not be modified.

Our study of the techniques for solving the coupled problem was motivated by the simulation of the interaction between channel flow and subsurface water flow. In particular, we have investigated realistic scenarios where the interface is composed of several patches belonging to different non-parallel planes and can not be reduced to one of the coordinate planes. The objective of our study is to develop an efficient and computationally cheap solution technique for the above-mentioned case.

We have implemented the Neumann-Dirichlet iterative matching and found that there are realistic applications where it is impossible to obtain the solution of the coupled problem due to very small convergence rates. The reduction of the coupled problem to the interface equation $[5,6]$ for fluxes seems to be technically impossible in the case of interface geometry under consideration [12]. Therefore, Krylov subspace methods are not applicable for the acceleration of the iterative coupling. To overcome this difficulty, we suggest another approach based on the recovery of the Neumann-Dirichlet iteration operator for the error. Being a direct method, the recovery requires as many pairs of coupling iterations as the number of interface degrees of freedom. It is independent of the value of the problem coefficients but requires higher precision for the iterative solution of the subproblems. The suggested method augments the flexibility of the Neumann-Dirichlet iteration allowing us to use different commercial codes as black box solvers. 
The recovery strategy appears to be applicable but expensive. An alternative method of solving the coupled problem is to form the interface equation for the normal stresses assuming continuous fluxes. This approach is capable of dealing with arbitrary interface geometries. Also, it suggests a simple way of iterative acceleration by Krylov subspace methods. In spite of the high stiffness of the interface operator, the number of GMRES iterations is just slightly sensitive to the value of coefficients [12]. This makes the latter approach very appealing in practical computations. In the 2D case and based on CG iterations, the same observation was made independently in [6]. We remark that forming the interface equation presumes certain modification of the source code of the subdomain solves. This reduces the flexibility of commercial software usage.

Another method suitable for realistic scenarios was suggested in [6]. It is based on the Robin-Robin domain decomposition method with two relaxation parameters for which Krylov subspace acceleration seems to be inapplicable.

\section{The differential formulation of the coupled problem}

Coupling of Darcy and Stokes flows corresponds to the situation in which an incompressible fluid contained in a domain $\Omega \subset \Re^{d}$ can flow both ways across the interface $\Gamma$. The interface separates two non-overlapping subdomains, $\Omega_{1}$ and $\Omega_{2}$, in which Stokes and Darcy flows are realized, respectively. Subdomain $\Omega_{1}$ represents the subdomain occupied by a fluid body, while the porous medium occupying $\Omega_{2}$ is assumed to be saturated with the fluid. We pose the coupled model as two differential problems whose solutions satisfy certain interface conditions. Find $\left(\mathbf{u}_{1}, p\right): \Omega_{1} \rightarrow \Re^{d} \times \Re$, and $\phi: \Omega_{2} \rightarrow \Re$, velocity, pressure and piezometric head, respectively, such that:

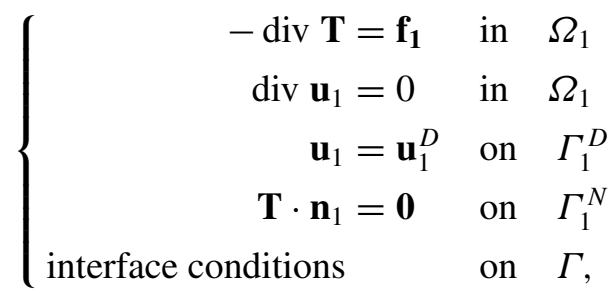

$$
\left\{\begin{array}{rlrl}
\operatorname{div} \epsilon \mathbf{u}_{2} & =f_{2} & & \text { in } \quad \Omega_{2} \\
\epsilon \mathbf{u}_{2} & =-\mathbf{K} \cdot \operatorname{grad} \phi & & \text { in } \quad \Omega_{2} \\
\phi & =\phi^{D} & & \text { on } \quad \Gamma_{2}^{D} \\
-\mathbf{n}_{2} \cdot \mathbf{K} \cdot \operatorname{grad} \phi & =\phi^{N} & & \text { on } \quad \Gamma_{2}^{N} \\
\text { interface conditions } & & \text { on } \quad \Gamma,
\end{array}\right.
$$


where $\Gamma_{1}=\overline{\Gamma_{1}^{D}} \cup \Gamma_{1}^{N}=\partial \Omega_{1} \backslash \Gamma, \Gamma_{2}=\overline{\Gamma_{2}^{D}} \cup \Gamma_{2}^{N}=\partial \Omega_{2} \backslash \Gamma$, and $\mathbf{n}_{i}$ is the outward unit normal to $\partial \Omega_{i}$.

Here, $\mathbf{T}$ is the stress tensor divided by the density, which may be linked to the rate of strain $\mathbf{D}\left(\mathbf{u}_{1}\right):=\frac{1}{2}\left[\operatorname{grad} \mathbf{u}_{1}+\operatorname{grad}^{T} \mathbf{u}_{1}\right]$ via the Newton constitutive relation as

$$
\mathbf{T}=-p \mathbf{I}+2 \nu \mathbf{D}\left(\mathbf{u}_{1}\right),
$$

where $p$ is the isotropic pressure divided by the density, $v$ the kinematic viscosity, $\epsilon$ the porosity, and $\mathbf{K}$ the conductivity tensor.

The set of interface conditions on $\Gamma$ is represented by three laws. The first interface condition is an expression for the dynamic equilibrium, in the form of a balance of normal forces across $\Gamma$. Let $\mathbf{t}=\mathbf{n}_{1} \cdot \rho \mathbf{T}$ be the traction exerted over the fluid. The traction $-\mathbf{t}$ exerted by the fluid over the interface must be compensated by the Darcy pressure $\rho g \phi$ :

$$
-\mathbf{t} \cdot \mathbf{n}_{1}=-\mathbf{n}_{1} \cdot \rho \mathbf{T} \cdot \mathbf{n}_{1}=\rho g \phi \quad \text { on } \quad \Gamma .
$$

Replacing $\mathbf{T}$ with the Newton constitutive relation yields

$$
p-2 \nu \mathbf{n}_{1} \cdot \mathbf{D}\left(\mathbf{u}_{1}\right) \cdot \mathbf{n}_{1}=g \phi \quad \text { on } \quad \Gamma .
$$

The second condition at the interface is the expression of mass conservation on $\Gamma$ yielding the conservation of the normal component of velocity:

$$
\mathbf{u}_{1} \cdot \mathbf{n}_{1}+\mathbf{u}_{2} \cdot \mathbf{n}_{2}=0 \quad \text { on } \quad \Gamma .
$$

The flow in the surface fluid body is uniquely defined provided that the tangential velocity at the interface is properly specified. The third interface condition is the Beavers-Joseph relation $[2,8]$ : the difference between the slip velocity of the free fluid $\mathbf{u}_{1} \cdot \tau_{j}$ and the tangential component of the seepage velocity $\mathbf{u}_{2} \cdot \tau_{j}$ was proposed to be proportional to the shear rate of the free fluid:

$$
\left(\mathbf{u}_{1}-\mathbf{u}_{2}\right) \cdot \tau_{j}=\frac{\sqrt{\left(\mathbf{K} \tau_{j}, \tau_{j}\right)}}{\alpha}\left(-\mathbf{n}_{1} \cdot \rho \mathbf{T}\right) \cdot \tau_{j},
$$

where $\tau_{j}, j=1, \ldots, d-1$, is the set of orthonormal tangent vectors on $\Gamma$.

Further simplification [14] of condition (6) is obtained by dropping out the term $\mathbf{u}_{2} \cdot \tau_{j}$ due to its negligible impact in the model. The interface condition, due to Beavers, Joseph, and Saffman, reads as follows:

$$
\mathbf{u}_{1} \cdot \tau_{j}=\frac{\sqrt{\left(\mathbf{K} \tau_{j}, \tau_{j}\right)}}{\alpha}\left(-\mathbf{n}_{1} \cdot \rho \mathbf{T}\right) \cdot \tau_{j} .
$$

This condition contributes an additional term to the bilinear form corresponding to the Stokes problem. In certain cases [3-6], condition (7) can be further simplified:

$$
-\mathbf{n}_{1} \cdot \rho \mathbf{T} \cdot \tau_{j}=0 .
$$


For instance, this simplification makes sense when $\alpha$ is very small. For a detailed justification of (8) we refer to [6]. We adopt the simple condition (8), since the more advanced condition (7) does not affect the objective of our research.

\section{Weak and finite element formulations}

Since the finite element approximation is based on the weak formulation, we rewrite the coupled differential problem in weak form. To do so, we define the spaces

$$
\begin{aligned}
H_{\Gamma_{1}} & :=\left\{v \in H^{1}\left(\Omega_{1}\right): v=0 \quad \text { on } \Gamma_{1}^{D}\right\}, \\
H_{1} & :=\left\{H_{\Gamma_{1}}\right\}^{d}, \\
Q & :=L_{2}\left(\Omega_{1}\right), \\
H_{2} & :=\left\{\psi \in H^{1}\left(\Omega_{2}\right): \psi=0 \text { on } \Gamma_{2}^{D}\right\}
\end{aligned}
$$

and specify functions $\phi_{e} \in H^{1}\left(\Omega_{2}\right), \phi_{e}=\phi^{D}$ on $\Gamma_{2}^{D}, \mathbf{u}_{e} \in\left\{H^{1}\left(\Omega_{1}\right)\right\}^{d}$, $\mathbf{u}_{e}=\mathbf{u}_{1}^{D}$ on $\Gamma_{1}^{D}$ so that the solution can be split according to

$$
\mathbf{u}_{1}=\mathbf{u}_{1}^{0}+\mathbf{u}_{e}, \mathbf{u}_{1}^{0} \in H_{1} \quad \phi=\phi_{0}+\phi_{e}, \phi_{0} \in H_{2} .
$$

Incorporating the interface conditions (4), (5), (8), we arrive at the weak formulation: Find $\mathbf{u}_{1}^{0} \in H_{1}, \phi_{0} \in H_{2}$ and $p \in Q$ such that:

$$
\begin{aligned}
& \int_{\Omega_{2}} \operatorname{grad} \psi \cdot \mathbf{K} \cdot \operatorname{grad} \phi_{0}-\int_{\Gamma} \epsilon \psi\left(\mathbf{u}_{1}^{0}+\mathbf{u}_{e}\right) \cdot \mathbf{n}_{1} \\
& =-\int_{\Omega_{2}} \operatorname{grad} \psi \cdot \mathbf{K} \cdot \operatorname{grad} \phi_{e}+\int_{\Gamma_{2}^{N}} \phi^{N} \psi+\int_{\Omega_{2}} f_{2} \psi \forall \psi \in H_{2}, \\
& \int_{\Omega_{1}} \nu \operatorname{grad} \mathbf{u}_{1}^{0} \cdot \operatorname{grad} \mathbf{w}-\int_{\Omega_{1}} p \operatorname{div} \mathbf{w}+\int_{\Gamma} g\left(\phi_{0}+\phi_{e}\right) \mathbf{w} \cdot \mathbf{n}_{1} \\
& =-\int_{\Omega_{1}} \nu \operatorname{grad} \mathbf{u}_{e} \cdot \operatorname{grad} \mathbf{w}+\int_{\Omega_{1}} \mathbf{f}_{1} \mathbf{w} \forall \mathbf{w} \in H_{1}, \\
& \int_{\Omega_{1}} q \operatorname{div} \mathbf{u}_{1}^{0}=-\int_{\Omega_{1}} q \operatorname{div} \mathbf{u}_{e} \forall q \in Q .
\end{aligned}
$$

Let $\Omega_{1, h}$ and $\Omega_{2, h}$ be conforming simplicial triangulations of the subdomains $\Omega_{1}, \Omega_{2}$ matching at the interface $\Gamma$. The finite element spaces are based on the meshes:

$$
\begin{aligned}
H_{\Gamma_{1}, h} & :=\left\{v_{h} \in C\left(\bar{\Omega}_{1}\right): v_{h}=0 \quad \text { on } \quad \Gamma_{1}^{D}, v_{h \mid E} \in \operatorname{isoP}_{2}(E) \forall E \in \Omega_{1, h}\right\}, \\
H_{1, h} & :=\left\{H_{\Gamma_{1}, h}\right\}^{d}, \\
Q_{h} & :=\left\{q_{h} \in C\left(\bar{\Omega}_{1}\right): q_{h \mid E} \in P_{1}(E) \forall E \in \Omega_{1, h}\right\}, \\
H_{2, h} & :=\left\{\psi_{h} \in C\left(\bar{\Omega}_{2}\right): \psi_{h}=0 \text { on } \Gamma_{2}^{D}, \psi_{h \mid E} \in P_{1}(E) \forall E \in \Omega_{2, h}\right\} .
\end{aligned}
$$


Let $\phi_{h}^{D}$ and $\mathbf{u}_{h}^{D}$ be the finite element analogs of $\phi_{e}$ and $\mathbf{u}_{e}$, respectively. They can be constructed by assigning the values of $\phi^{D}, \mathbf{u}_{1}^{D}$ to the boundary nodes on $\Gamma_{2}^{D}$ and $\Gamma_{1}^{D}$ respectively, and zero values to all other nodes of the mesh. Therefore, the following splitting is feasible:

$$
\mathbf{u}_{1 h}=\mathbf{u}_{1 h}^{0}+\mathbf{u}_{h}^{D}, \mathbf{u}_{1 h}^{0} \in H_{1, h} \quad \phi_{h}=\phi_{0 h}+\phi_{h}^{D}, \phi_{0 h} \in H_{2, h} .
$$

The coupled FEM problem can be stated as follows. Find $\mathbf{u}_{1 h}^{0} \in H_{1, h}$, $\phi_{0 h} \in H_{2, h}$ and $p_{h} \in Q_{h}$ such that

$$
\begin{gathered}
\int_{\Omega_{2}} \operatorname{grad} \psi_{h} \cdot \mathbf{K} \cdot \operatorname{grad} \phi_{0 h}-\int_{\Gamma} \epsilon \psi_{h}\left(\mathbf{u}_{1 h}^{0}+\mathbf{u}_{h}^{D}\right) \cdot \mathbf{n}_{1}=\int_{\Gamma_{2}^{N}} \phi^{N} \psi_{h}+ \\
-\int_{\Omega_{2}} \operatorname{grad} \psi_{h} \cdot \mathbf{K} \cdot \operatorname{grad} \phi_{h}^{D}+\int_{\Omega_{2}} f_{2} \psi_{h} \quad \forall \psi_{h} \in H_{2, h}, \\
\int_{\Omega_{1}} \nu \operatorname{grad} \mathbf{u}_{1 h}^{0} \cdot \operatorname{grad} \mathbf{w}_{h}-\int_{\Omega} p_{h} \operatorname{div} \mathbf{w}_{h}+\int_{\Gamma} g\left(\phi_{0 h}+\phi_{h}^{D}\right) \mathbf{w}_{h} \cdot \mathbf{n}_{1}= \\
-\int_{\Omega_{1}} \nu \operatorname{grad} \mathbf{u}_{h}^{D} \cdot \operatorname{grad} \mathbf{w}_{h}+\int_{\Omega_{1}} \mathbf{f}_{1} \mathbf{w}_{h} \quad \forall \mathbf{w}_{h} \in H_{1, h}, \\
\int_{\Omega_{1}} q_{h} \operatorname{div} \mathbf{u}_{1 h}^{0}=-\int_{\Omega_{1}} q_{h} \operatorname{div} \mathbf{u}_{h}^{D} \quad \forall q_{h} \in Q_{h} .
\end{gathered}
$$

We refer to $\Omega_{1, h / 2}$ as the triangulation obtained by splitting each tetrahedron from $\Omega_{1, h}$ into eight subtetrahedra. Further, we denote by $U_{0}$ and $U_{\Gamma}$ the vectors whose components are the values of $\mathbf{u}_{1 h}^{0}$ at the nodes of $\Omega_{1, h / 2} \backslash\left(\Gamma \cup \bar{\Gamma}_{1}^{D}\right)$ and of $\mathbf{u}_{1 h}^{0}$ at the interface nodes of $\Omega_{1, h / 2} \backslash \bar{\Gamma}_{1}^{D}$. Also $P$ stands for the vector of the values of the fluid pressure at the nodes of $\Omega_{1, h}$. In addition, $\Phi_{0}$ contains the values of the piezometric head $\phi_{0 h}$ at the nodes of $\Omega_{1, h} \backslash\left(\Gamma \cup \bar{\Gamma}_{2}^{D}\right)$, and $\Phi_{\Gamma}$ those at the nodes on $\Gamma \backslash \bar{\Gamma}_{2}^{D}$. The algebraic representation of the coupled problem (13)-(15) is

$$
\left[\begin{array}{ccccc}
A_{0} & A_{0 \Gamma} & B_{0}^{T} & 0 & 0 \\
A_{0 \Gamma}^{T} & A_{\Gamma} & B_{\Gamma}^{T} & P_{\mathbf{n}_{\Gamma}}^{T} M_{\Phi_{\Gamma}} & 0 \\
B_{0} & B_{\Gamma} & 0 & 0 & 0 \\
0 & -M_{\lambda} P_{\mathbf{n}_{\Gamma}} & 0 & D_{\Gamma} & D_{0 \Gamma}^{T} \\
0 & 0 & 0 & D_{0 \Gamma} & D_{0}
\end{array}\right]\left[\begin{array}{c}
U_{0} \\
U_{\Gamma} \\
P \\
\Phi_{\Gamma} \\
\Phi_{0}
\end{array}\right]=\left[\begin{array}{c}
F_{0} \\
F_{\Gamma} \\
F_{p} \\
G_{\Gamma} \\
G_{0}
\end{array}\right] .
$$

The main difference between the system (16) and its counterpart from [5,6] is with respect to $U_{\Gamma}$. In our setting, $U_{\Gamma}$ contains three velocity components at each interface node, whereas in [5,6] $U_{\Gamma}$ is composed of only the normal component. This allows us to treat arbitrary interfaces rather than interfaces whose normal is orthogonal to a coordinate plane.

The main objective of the paper is to discuss several approaches to the iterative solution of (16) under the assumption that iterative solvers for the 
discrete Stokes and Darcy problems are available. The stiffness of the matrix of (16) is affected by the mesh size $h$, the scalar coefficients $v, \epsilon, g$ and the tensor $\mathbf{K}$. Therefore, the iterative solution is sensitive to these parameters as well, and the comparison of different approaches should take this sensitivity into account.

\section{Iterative solution procedures}

\subsection{Neumann-Dirichlet iterations}

The Neumann-Dirichlet algorithm [3,4] replaces the tightly coupled problems (13)-(15) by an iterative coupling process which converges to the solution of (13)-(15). In physical terms, the Neumann-Dirichlet iteration is matching the interface fluxes keeping the normal stresses continuous. In algebraic terms, the Neumann-Dirichlet iteration for the solution of (16) is defined as follows. Let $\lambda^{k}=P_{\mathbf{n}_{\Gamma}} U_{\Gamma}^{k}$ denote the $k$ th iterate with entries associated with the nodes of $\left(\Omega_{1, h} \backslash \bar{\Gamma}_{1}^{D}\right) \cap \Gamma$.

Step 1: Initialization. Choose an initial guess $\lambda^{0}$ and the relaxation parameter $\theta$.

Step 2: Iteration Loop. For $k \geq 0$

1. compute $\left[\begin{array}{l}\Phi_{\Gamma}^{k+1} \\ \Phi_{0}^{k+1}\end{array}\right]$ such that

$$
\left[\begin{array}{cc}
D_{\Gamma} & D_{0 \Gamma}^{T} \\
D_{0 \Gamma} & D_{0}
\end{array}\right]\left[\begin{array}{c}
\Phi_{\Gamma}^{k+1} \\
\Phi_{0}^{k+1}
\end{array}\right]=\left[\begin{array}{c}
G_{\Gamma}+M_{\lambda} \lambda^{k} \\
G_{0}
\end{array}\right] ;
$$

2. compute $\left[\begin{array}{c}U_{0}^{k+1} \\ U_{\Gamma}^{k+1} \\ P^{k+1}\end{array}\right]$ such that

$$
\left[\begin{array}{ccc}
A_{0} & A_{0 \Gamma} & B_{0}^{T} \\
A_{0 \Gamma}^{T} & A_{\Gamma} & B_{\Gamma}^{T} \\
B_{0} & B_{\Gamma} & 0
\end{array}\right]\left[\begin{array}{c}
U_{0}^{k+1} \\
U_{\Gamma}^{k+1} \\
P^{k+1}
\end{array}\right]=\left[\begin{array}{c}
F_{0} \\
F_{\Gamma}-P_{\mathbf{n}_{\Gamma}}^{T} M_{\Phi_{\Gamma}} \Phi_{\Gamma}^{k+1} \\
F_{p}
\end{array}\right]
$$

3. update $\lambda^{k}$ according to

$$
\lambda^{k+1}=\theta P_{\mathbf{n}_{\Gamma}} U_{\Gamma}^{k+1}+(1-\theta) \lambda^{k} .
$$


The realization of the substeps (17)-(18) requires the iterative solution of a symmetric positive definite and of a saddle point systems, respectively. The system (17) is solved by the conjugate gradient method preconditioned by a V-cycle of an algebraic multigrid [16] which provides linear arithmetic complexity and a convergence rate independent of the mesh size. The system (18) is solved by the generalized minimum residual method although the Lanczos method of minimal iterations is applicable as well. The preconditioner in the latter case is a block diagonal matrix. Each block corresponding to a velocity component is represented by the $\mathrm{V}$-cycle of the algebraic multigrid method. The pressure block corresponds to a few iterations with Chebyshev parameters solving approximately a system with the mass matrix.

The Neumann-Dirichlet algorithm has advantages and drawbacks. It benefits from its simplicity of implementation and a simple and straightforward way of choosing the initial guess in the iterative solution of the subproblems. As we see below, this saves a lot of computational work. On the other hand, the convergence rate of Neumann-Dirichlet iterations is very sensitive to the problem coefficients. Also, the choice of the parameter $\theta$ may be cumbersome: overly large values eventually result in divergence, and overly small values result in non-optimal convergence rates.

The sensitivity of the convergence rate and of $\theta$ with respect to the problem parameters is critical in realistic applications. When $U_{\Gamma}$ only represents the normal to the interface component of the velocity, the analysis in [4,6] suggests that $\theta \in\left[0, \theta_{\mathrm{MAX}}\right]$, where, for $v / \epsilon^{2} \ll g /\|\mathbf{K}\|$, one has $\theta_{\text {MAX }} \sim \mathcal{O}\left(\epsilon^{5} v^{2}\|\mathbf{K}\|^{2} / g^{2}\right)$. For $\theta_{\text {MAX }}=o(1)$ the convergence rate is $1-C \theta_{\mathrm{MAX}}$. Therefore, the smaller $\theta_{\mathrm{MAX}}$ is, the worse is the observed convergence rate. Although an experimental evaluation of $\theta_{\text {MAX }}$ is less pessimistic, $\theta_{\text {MAX }} \sim \mathcal{O}(\nu \mathbf{K} /(\epsilon g))$, for realistic values of conductivity $\|\mathbf{K}\| \sim 10^{-5}$, porosity $\epsilon \sim 10^{-1}$ (the case of limestone) and kinematic viscosity $v \sim 1$ (the case of water), $g \sim 10$, the empirical value of $\theta_{\operatorname{MAX}}$ is $10^{-11}$. This renders the method useless due to an unacceptable rate of convergence.

\subsection{Recovery of the iteration operator for the error}

An alternative technique is based on the recovery of the Neumann-Dirichlet iteration operator for the error. The idea behind it is simple: consider three iterative guesses $\lambda^{0}, \lambda^{1}$ and $\lambda^{2}$ to the interface solution $\lambda=P_{\mathbf{n}_{\Gamma}} U_{\Gamma}$ of our problem. Then, due to the linearity of the iteration operator $T_{\Lambda}$ we have

$$
\lambda-\lambda^{1}=T_{\Lambda}\left(\lambda-\lambda^{0}\right), \lambda-\lambda^{2}=T_{\Lambda}\left(\lambda-\lambda^{1}\right)
$$

and

$$
\lambda^{2}-\lambda^{1}=T_{\Lambda}\left(\lambda^{1}-\lambda^{0}\right) .
$$


Eliminating $\lambda^{2}$ in (20), we obtain the following equation for $\lambda$ :

$$
\left(I-T_{\Lambda}\right) \lambda=\lambda_{h}^{1}-T_{\Lambda} \lambda^{0},
$$

which suggests the following procedure.

1. For every node on the interface $\Gamma \backslash \Gamma_{1}^{D}$, indicated by $k=1, \ldots, N$, take $\lambda_{k}^{0}=e_{k}$, which is the $k$ th unit vector in $\Re^{N}$ and find $\lambda_{k}^{1}$ and $\lambda_{k}^{2}$ performing two steps of (17)-(19).

2. Form the matrices $T_{01}$ and $T_{12}$ with columns $\lambda_{k}^{0}-\lambda_{k}^{1}$ and $\lambda_{k}^{1}-\lambda_{k}^{2}$, respectively.

3. Recover the iteration operator $T_{\Lambda}=T_{12} \cdot T_{01}^{-1}$.

4. Recover the solution $\lambda_{h}=\left(I-T_{\Lambda}\right)^{-1}\left(\lambda_{k}^{1}-T_{\Lambda} \lambda_{k}^{0}\right)$ for an arbitrary $k$.

In practice, the cost of this procedure is dominated by the construction of the matrices $T_{01}$ and $T_{12}$. In effect, the construction of a pair of columns corresponding to a node at the interface implies two Neumann-Dirichlet iterations in order to obtain $\lambda^{1}$ and $\lambda^{2}$. Each iteration consists of solving a Stokes problem and a Darcy problem. The order $N$ of the matrix $T_{\Lambda}$ depends on the geometry of the subdomains and on the mesh properties. In the case of a uniform grid for the unit cube partitioned into two equal subdomains by a plane, $N \approx h^{-2}$, the number of nodes in the subdomains is $h^{-3} / 2$, and hence the cost of forming $T_{01}, T_{12}$ is $2 N \log \varepsilon^{-1} \mathcal{O}\left(N^{1.5}\right)$. Here, $\varepsilon$ is the norm residual tolerance for both the Stokes and the Darcy subproblems. We note that, although the asymptotic arithmetic complexity of the inversion of the dense matrix $T_{01}$ and the solution of the dense system (22), $\mathcal{O}\left(N^{3}\right)$, is higher than $\varepsilon^{-1} \mathcal{O}\left(N^{2.5}\right)$, the practical restriction for $N \lesssim 10^{3}$ makes the cost of steps 3 and 4 negligible compared to the cost of step 1 .

Being a "direct" method for solving the system (16), the procedure "converges" exactly for $N$ pairs of Neumann-Dirichlet iterations. However, the procedure discussed above may be rather expensive if $N$ is more than a few thousands. Moreover, in realistic scenarios the stiffness of the matrix $T_{01}$ is very high. Therefore, the entries of $T_{01}$ must be computed very accurately. To this end, the value of the tolerance $\varepsilon$ must be very small which requires a larger number of iterations for the subproblems.

\subsection{Iterative solution of the interface equation}

Let $C$ denote the matrix block of (16) given by

$$
C=\left[\begin{array}{ccc}
A_{0} & A_{0 \Gamma} & B_{0}^{T} \\
A_{0 \Gamma}^{T} & A_{\Gamma} & B_{\Gamma}^{T} \\
B_{0} & B_{\Gamma} & 0
\end{array}\right] .
$$


In order to write the interface equation, we consider the first three equations of the system (16):

$$
C\left[\begin{array}{c}
U_{0} \\
U_{\Gamma} \\
P
\end{array}\right]+\left[\begin{array}{c}
0 \\
P_{\mathbf{n}_{\Gamma}}^{T} M_{\Phi_{\Gamma}} \Phi_{\Gamma} \\
0
\end{array}\right]=\left[\begin{array}{c}
F_{0} \\
F_{\Gamma} \\
F_{p}
\end{array}\right] .
$$

The matrix $C$ corresponds to the standard discrete Stokes problem and is invertible so that

$$
\left[\begin{array}{c}
U_{0} \\
U_{\Gamma} \\
P
\end{array}\right]=C^{-1}\left[\begin{array}{c}
F_{0} \\
F_{\Gamma} \\
F_{p}
\end{array}\right]-C^{-1}\left[\begin{array}{c}
0 \\
P_{\mathbf{n}_{\Gamma}}^{T} M_{\Phi_{\Gamma}} \Phi_{\Gamma} \\
0
\end{array}\right] .
$$

From the last equation of (16) we eliminate $\Phi_{0}$ :

$$
\Phi_{0}=D_{0}^{-1}\left(G_{0}-D_{0_{\Gamma}} \Phi_{\Gamma}\right) .
$$

The fourth equation implies that

$$
\begin{aligned}
{\left[0-M_{\lambda} P_{\mathbf{n}_{\Gamma}} 0\right] } & \left(C^{-1}\left[\begin{array}{c}
F_{0} \\
F_{\Gamma} \\
F_{p}
\end{array}\right]-C^{-1}\left[\begin{array}{c}
0 \\
P_{\mathbf{n}_{\Gamma}}^{T} M_{\Phi_{\Gamma}} \Phi_{\Gamma} \\
0
\end{array}\right]\right) \\
& +D_{\Gamma} \Phi_{\Gamma}+D_{0_{\Gamma}}^{T} D_{0}^{-1}\left(G_{0}-D_{0_{\Gamma}} \Phi_{\Gamma}\right)=G_{\Gamma} .
\end{aligned}
$$

Defining $S_{\mathcal{D}}^{h}=D_{\Gamma}-D_{0_{\Gamma}}^{T} D_{0}^{-1} D_{0_{\Gamma}}$, we obtain

$$
\begin{aligned}
{\left[\begin{array}{ll}
0 M_{\lambda} P_{\mathbf{n}_{\Gamma}} 0
\end{array}\right] C^{-1}\left[\begin{array}{c}
0 \\
P_{\mathbf{n}_{\Gamma}}^{T} M_{\Phi_{\Gamma}} \Phi_{\Gamma} \\
0
\end{array}\right]+S_{\mathcal{D}}^{h} \Phi_{\Gamma} } \\
\quad=G_{\Gamma}-D_{0 \Gamma}^{T} D_{0}^{-1} G_{0}+\left[\begin{array}{ll}
0 M_{\lambda} P_{\mathbf{n}_{\Gamma}} & 0
\end{array}\right] C^{-1}\left[\begin{array}{c}
F_{0} \\
F_{\Gamma} \\
F_{p}
\end{array}\right]
\end{aligned}
$$

Setting $\mathcal{J}:=G_{\Gamma}-D_{0 \Gamma}^{T} D_{0}^{-1} G_{0}+\left[0 M_{\lambda} P_{\mathbf{n}_{\Gamma}} 0\right] C^{-1}\left[\begin{array}{lll}F_{0} & F_{\Gamma} & F_{p}\end{array}\right]^{T}$ and $\mathcal{C}^{-1}=\left[\begin{array}{lll}0 & I & 0\end{array}\right] C^{-1}\left[\begin{array}{lll}0 & I & 0\end{array}\right]^{T}$, we arrive at

$$
\left(S_{\mathcal{D}}^{h}+M_{\lambda} P_{\mathbf{n}_{\Gamma}} \mathcal{C}^{-1} P_{\mathbf{n}_{\Gamma}}^{T} M_{\Phi_{\Gamma}}\right) \Phi_{\Gamma}=\mathcal{J}
$$

or, equivalently,

$$
\left(I+\left(S_{\mathcal{D}}^{h}\right)^{-1} M_{\lambda} P_{\mathbf{n}_{\Gamma}} \mathcal{C}^{-1} P_{\mathbf{n}_{\Gamma}}^{T} M_{\Phi_{\Gamma}}\right) \Phi_{\Gamma}=\left(S_{\mathcal{D}}^{h}\right)^{-1} \mathcal{J} .
$$

We consider Richardson's iteration for (28):

$$
\Phi_{\Gamma}^{k+1}=\Phi_{\Gamma}^{k}+\theta\left(\left(S_{\mathcal{D}}^{h}\right)^{-1} \mathcal{J}-\left(I+\left(S_{\mathcal{D}}^{h}\right)^{-1} M_{\lambda} P_{\mathbf{n}_{\Gamma}} \mathcal{C}^{-1} P_{\mathbf{n}_{\Gamma}}^{T} M_{\Phi_{\Gamma}}\right) \Phi_{\Gamma}^{k}\right)
$$


The physical meaning of the Richardson iteration is matching normal stresses under the assumption of flux continuity. The use of the unknown iterative parameter $\theta$ is not necessary, if one switches to Krylov subspace iterations. For instance, it can be solved by CG iterations [6]. We suggest solving (28) by GMRES keeping in mind the stability of GMRES iterations. As we show below, the use of Krylov subspace iterations is very efficient, since the number of iterations is less sensitive to the stiffness of the system matrix determined by the magnitude of the coefficients.

An alternative way of deriving an interface equation is to eliminate $U_{0}$, $P, \Phi_{0}, \Phi_{\Gamma}$ instead of eliminating $U_{0}, U_{\Gamma}, P, \Phi_{0}$ :

$$
\left(S_{\mathcal{S}}^{h}+P_{\mathbf{n}_{\Gamma}}^{T} M_{\Phi_{\Gamma}}\left(S_{\mathcal{D}}^{h}\right)^{-1} M_{\lambda} P_{\mathbf{n}_{\Gamma}}\right) U_{\Gamma}=F_{S}-P_{\mathbf{n}_{\Gamma}}^{T} M_{\Phi_{\Gamma}}\left(S_{\mathcal{D}}^{h}\right)^{-1} G_{S},
$$

where

$$
\begin{gathered}
F_{S}=F_{\Gamma}-\left[\begin{array}{cc}
A_{0 \Gamma}^{T} & B_{\Gamma}^{T}
\end{array}\right]\left[\begin{array}{cc}
A_{0} & B_{0}^{T} \\
B_{0} & 0
\end{array}\right]^{-1}\left[\begin{array}{c}
F_{0} \\
F_{p}
\end{array}\right], \quad G_{S}=G_{\Gamma}-D_{0 \Gamma}^{T} D_{0}^{-1} G_{0}, \\
S_{\mathcal{S}}^{h}=A_{\Gamma}-\left[\begin{array}{ll}
A_{0 \Gamma}^{T} & B_{\Gamma}^{T}
\end{array}\right]\left[\begin{array}{cc}
A_{0} & B_{0}^{T} \\
B_{0} & 0
\end{array}\right]^{-1}\left[\begin{array}{c}
A_{0 \Gamma} \\
B_{\Gamma}
\end{array}\right] .
\end{gathered}
$$

The physical meaning of the alternative interface equation is matching fluxes under the assumption of normal stress continuity. We note that the derivation of (29) is confined to the case when the vector $F_{S}$ is available. This is the case if $U_{\Gamma}$ represents only the normal component. Then, the matrix $\left[\begin{array}{cc}A_{0} & B_{0}^{T} \\ B_{0} & 0\end{array}\right]$ is the discretization of the Stokes operator with homogeneous natural boundary condition on $\Gamma$ and inherited boundary conditions on $\partial \Omega_{1} \backslash \Gamma$, which are compatible [13]. Therefore, the system

$$
\left[\begin{array}{cc}
A_{0} & B_{0}^{T} \\
B_{0} & 0
\end{array}\right]\left[\begin{array}{c}
\tilde{U}_{0} \\
\tilde{P}
\end{array}\right]=\left[\begin{array}{c}
F_{0} \\
F_{p}
\end{array}\right]
$$

is compatible and $F_{S}$ may be found. Moreover, in this case Eq. (29) can be transformed to the scalar equation

$$
\begin{aligned}
\left(I+P_{\mathbf{n}_{\Gamma}}\left(S_{\mathcal{S}}^{h}\right)^{-1} P_{\mathbf{n}_{\Gamma}}^{T} M_{\Phi_{\Gamma}}\left(S_{\mathcal{D}}^{h}\right)^{-1} M_{\lambda}\right) \mu & \\
& =P_{\mathbf{n}_{\Gamma}}\left(S_{\mathcal{S}}^{h}\right)^{-1}\left(F_{S}-P_{\mathbf{n}_{\Gamma}}^{T} M_{\Phi_{\Gamma}}\left(S_{\mathcal{D}}^{h}\right)^{-1} G_{S}\right) .
\end{aligned}
$$

The assignment of normal components to $U_{\Gamma}$ confines the set of possible interfaces to patches on a coordinate plane which is not adequate for many practical problems. 
We now consider the general case in which $U_{\Gamma}$ represents all velocity components, and verify the compatibility of (30). In this case, $\left[\begin{array}{cc}A_{0} & B_{0}^{T} \\ B_{0} & 0\end{array}\right]$ represents the discretization of the Stokes operator with homogeneous Dirichlet boundary condition on $\Gamma$. Hence, the condition $F_{p} \in \operatorname{Im} B_{0}$ has to be verified. Recall that from (16) it follows that there exists $U_{0}$ such that $F_{p}-B_{\Gamma} U_{\Gamma}=B_{0} U_{0}$, i.e., $F_{p}-B_{\Gamma} U_{\Gamma} \in \operatorname{Im} B_{0}$. Therefore, $F_{p} \in \operatorname{Im} B_{0}$ if and only if $B_{\Gamma} U_{\Gamma} \in \operatorname{Im} B_{0}$, i.e., if there exists $W_{0}$ such that $B_{\Gamma} U_{\Gamma}=B_{0} W_{0}$. By the definition of $B_{0}$ and $B_{\Gamma}$,

$$
\begin{aligned}
& \left(B_{0} W_{0}, q\right)=\int_{\Omega_{1}} q_{h} \operatorname{div} \mathbf{W}_{0}^{h}=\int_{\Gamma_{1}^{N}} q_{h}\left(\mathbf{n}_{\Gamma} \cdot \mathbf{W}_{0}^{h}\right) \\
& \left(B_{\Gamma} W_{\Gamma}, q\right)=\int_{\Omega_{1}} q_{h} \operatorname{div} \mathbf{U}_{\Gamma}^{h}=\int_{\Gamma} q_{h}\left(\mathbf{n}_{\Gamma} \cdot \mathbf{U}_{\Gamma}^{h}\right)
\end{aligned}
$$

for any $q_{h}$ from $Q_{h}$. Here, $\mathbf{W}_{0}^{h}$ and $\mathbf{U}_{\Gamma}^{h}$ denote the finite element extensions of the vectors $W_{0}$ and $U_{\Gamma}$, respectively. Therefore,

$$
\left(B_{0} W_{0}-B_{\Gamma} W_{\Gamma}, q\right)=\int_{\Gamma_{1}^{N}} q_{h}\left(\mathbf{n}_{\Gamma} \cdot \mathbf{W}_{0}^{h}\right)-\int_{\Gamma} q_{h}\left(\mathbf{n}_{\Gamma} \cdot \mathbf{U}_{\Gamma}^{h}\right) \quad \forall q_{h} \in Q_{h} .
$$

Since $\mathbf{W}_{0}^{h}=0$ on $\Gamma, \mathbf{U}_{\Gamma}^{h}=0$ on $\Gamma_{1}^{N}$, the left-hand side of (32) can not vanish for any $q$. From this observation we conclude that the only way to derive the interface equation seems to be the approach resulting in (28).

\section{Numerical experiments}

The above approaches have been implemented for two model 3D problems. The first problem is the simplest: the interface $\Gamma$ is planar and all the coefficients are set to 1 . The second problem is more complicated due to a non-planar interface and realistic coefficients. In both cases, the numerical experiments were performed on a Pentium $4(2.5 \mathrm{GH})$. The details of the iterative solvers for the subproblems were discussed in Sect. 4.1.

\section{Model problem with an analytic solution}

First, we consider a problem with an analytic solution and unit coefficients. Let $\Omega_{1}=(0,1)^{2} \times(1,2), \Omega_{2}=(0,1)^{3}, \Gamma=\{z=1\} \cap \bar{\Omega}_{1}, \Gamma_{1}^{N}=\emptyset$, 
$\Gamma_{2}^{N}=\{y=0$ or $y=1\} \cap \bar{\Omega}_{2}, \mathbf{K}=k \mathbf{I}$, and $k=\epsilon=v=g=1$. The functions [5],

$$
\begin{aligned}
& \mathbf{u}_{1}=\left(\begin{array}{c}
-\cos (\pi x / 2) \sin (\pi z / 2)+1 \\
0 \\
\sin (\pi x / 2) \cos (\pi z / 2)-3
\end{array}\right) \\
& p=-(2 / \pi+\pi / 2) \sin (\pi x / 2) \sin (\pi z / 2)+3 z \\
& \phi=-2 / \pi \sin (\pi x / 2) \sin (\pi z / 2)+3 z,
\end{aligned}
$$

represent the solution of the coupled problem (9)-(11) with

$$
\begin{aligned}
& \mathbf{f}_{1}=\left(\begin{array}{c}
-\left(0.75 \pi^{2}+1\right) \cos (\pi x / 2) \sin (\pi z / 2) \\
0 \\
\left(0.25 \pi^{2}-1\right) \sin (\pi x / 2) \cos (\pi z / 2)+3
\end{array}\right) \\
& f_{2}=-\pi \sin (\pi x / 2) \sin (\pi z / 2) .
\end{aligned}
$$

A sequence of uniform meshes was generated by subsequent refinement of each tetrahedron of a coarse tetrahedral mesh. The latter was obtained by partitioning of the unit cube $(0,1)^{3}$ into 8 sub-cubes, splitting each sub-cube into 6 tetrahedra and mapping the mesh into $(0,1)^{2} \times(0,2)$.

The basic features of the iterative process (17)-(19) as well as its arithmetic complexity are reported in Table 1 . The residual tolerance for the subproblems (17), (18) was chosen to be $10^{-7}$ and $10^{-6}$, respectively. The coupling iterations were terminated when $\left\|\lambda^{k+1}-\lambda^{k}\right\| \leq 10^{-4}\left\|\lambda^{k}\right\|$. Here, \#ND denotes the number of coupling iterations, $\# \mathrm{G}_{k} \#\left(\mathrm{P}_{k}\right)$ stands for the number of GMRES (PCG) iterations for the Stokes (Darcy) subproblem performed at the $k$ th coupling iteration, $\mathrm{CPU} \mathrm{itS}_{\mathrm{S}}\left(\mathrm{CPU}_{\mathrm{itD}}\right)$ refers to the execution time of one GMRES (PCG) iteration, and $\mathrm{CPU}_{\text {tot }}$ denotes the total execution time. As can be seen from Table 1, the number of coupling iterations does not depend on the mesh size, whereas the numbers of the GMRES and the PCG iterations decrease in the course of coupling iterations, due to better initial guesses. We note that the convergence rate of the GMRES iterations saturates as $h \rightarrow 0$. The boundedness of the number of PCG iterations cannot be clearly observed. We explain the latter phenomenon by

Table 1. Performance of iterative solvers for a sequence of refined meshes

\begin{tabular}{c|c|c|c|c|c|c|c|c}
\hline Mesh size & $\# \mathrm{ND}$ & $\# \mathrm{G}_{1}$ & $\# \mathrm{P}_{1}$ & $\#_{15}$ & $\# \mathrm{P}_{15}$ & $\mathrm{CPU}_{\mathrm{itS}}$ & $\mathrm{CPU}_{\mathrm{itD}}$ & $\mathrm{CPU}_{\text {tot }}$ \\
\hline$h=1 / 8$ & 15 & 55 & 5 & 2 & 4 & 0.04 & $<0.01$ & 12.7 \\
$h=1 / 16$ & 15 & 60 & 9 & 3 & 3 & 0.65 & 0.015 & 204 \\
$h=1 / 32$ & 15 & 62 & 12 & 3 & 3 & 6.5 & 0.17 & 2108 \\
\hline
\end{tabular}


the extremely high convergence rate of the PCG for small Darcy problems. On the other hand, the cost per iteration (for both GMRES and PCG) and the total CPU time are linear with respect to the number of unknowns (each refinement results in an 8-fold increase of the number of unknowns). The Neumann-Dirichlet iterations is apparently a very efficient solution method for (16) in this case.

\section{Interaction of a channel and a porous medium}

Secondly, we consider the penetration of fluid from a 3D channel with a solid top lid and a sealed outlet into porous media. Two cases of porous material are considered, limestone and coarse sand (instead of limestone, one may consider sandstone or dolomite, and coarse sand may be substituted with gravel or karstic limestone or permeable basalt). In the international system of units, the problem coefficients are $\mathbf{K}=10^{-5} \mathbf{I}$ for limestone, $\mathbf{K}=10^{-2} \mathbf{I}$ for sand, $\epsilon=0.1, g=10$. We consider fluid with high viscosity $v=1$ (for water $v=10^{-6}$ ), since small values of $v$ make the system (16) so stiff that it is non-tractable in conventional double precision arithmetics. We note that $v \ll 1$ does not affect the efficiency of the solvers except for the NeumannDirichlet iterations. The large value $v=1$ is set for the sake of presentation of the simulation. Let $\Omega_{1}=(0,40) \times(8.175,11.825) \times(8.175,10)$ and $\Omega_{2}=(0,40) \times(0,20) \times(0,10) \backslash \Omega_{1}$. Moreover, $\Gamma_{1}^{D}$ consists of the inlet $(x=0)$, the outlet $(x=40)$ and the top $(z=10)$ of $\Omega_{1} ; \Gamma_{2}^{D}$ is the bottom face of the domain $\Omega_{2}$. At the inlet of the channel, the $x$-component of the inflow is $u_{x}=(11.825-y)(y-8.175)(10-z)(z-8.175) 4 / 1.825^{4}$. All other Dirichlet conditions are homogeneous. The Neumann boundary conditions for $\Omega_{2}$ are set to zero. The right-hand sides representing sources are set to zero, $\mathbf{f}_{\mathbf{1}}=\mathbf{0}, f_{2}=0$. This implies that all the fluid entering the channel through the inlet infiltrates into the porous media and leaves it through the bottom face.

The mesh sequence is formed from tetrahedral partitionings of rectangular grids with $2^{k}$ steps in the $x$ and $y$ directions, and $2^{k-1}$ steps in the $z$ direction, $k=4,5,6$. For $k=5$ both the Stokes and the Darcy systems have approximately 20000 unknowns, and for $k=6$ they have about 150000 unknowns.

Figures 1, 2, 3 show cross-sections of the Stokes and the Darcy velocity fields at the planes $y=10$ and $z=9$. The computed Darcy velocity vectors (Figs. 2,3) are normal to the interface and are constant along the $x$-axis in the case of limestone, whereas they are not normal to the interface and not constant along $x$-axis in the case of coarse sand. The Stokes velocity (Fig. 1) field exhibits a small decrease in magnitude along the $x$-axis and a boundary layer at the plane $x=40$ in the case of limestone, whereas in the case of coarse sand it shows a linear decrease in magnitude along the $x$-axis, up to 
vanishing at the plane $x=40$. Due to inconsistency of boundary conditions, a singularity of the flow fields is observed at the plane $x=0$.

The basic reason for such a different behavior of the solution of the coupled problem is the conductivity of the porous material. Indeed, in the case of sand, the fluid easily penetrates the interface, and almost all the injected water can infiltrate the vicinity of the inlet. On the other hand, the flow in the Stokes region accommodates itself to fit the conservation of mass. The other part of the domain is occupied by almost stagnant fluid. In the case of limestone, the infiltration is not strong near the inlet and is more uniformly distributed along the interface. The uniformity of the infiltration into the Darcy region and the symmetry of boundary conditions result in the orthogonality of Darcy velocities to the interface. In the presence of an impervious boundary condition, the conservation of mass promotes a flushing flow along the interface, additional to the one normal to the interface. The module of velocity of the Stokes flow is dominated by the longitudinal component. In accordance with conservation of mass, the above-mentioned excess of injection into the sand results in a stronger decrease of longitudinal velocity along the channel when compared with that in the case of limestone. In both cases, the solution vanishes at the end of the channel in accordance with the boundary condition. This, together with the higher longitudinal velocity, explains the formation of a stronger boundary layer in the case of limestone.

The two cases are very different not only in the solutions, but in the solution procedures as well. The sand case was handled by the NeumannDirichlet iteration with $\theta=0.01$. For the intermediate grid $(k=5), 683$ iterations and 4144 seconds were required to obtain $\left\|\lambda^{k+1}-\lambda^{k}\right\| \leq 10^{-4}\left\|\lambda^{k}\right\|$. The case of limestone can not be treated in a similar way: the value $\theta=10^{-5}$ makes the Neumann-Dirichlet iterations inapplicable. However, the recovery of the iteration operator readily solves the problem regardless of the value $\|\mathbf{K}\|$. Since the number of interface degrees of freedom for $\lambda$ in the mesh considered is 341 , the recovery requires 341 pairs of iterations (17)-(19), one pair for each $\lambda_{k}, k=1, \ldots, 341$. Accidentally, the total number of the coupled iterations was also 683 in this case. However, it took 15179 seconds, that is, almost 4 times slower than the Neumann-Dirichlet iterations for the case of sand. The reason is two-fold: first, the residual tolerance for both

Fig. 1. $X Z$ cross-section of isosurfaces of Stokes velocity module, in the case of sand (bottom) and of limestone (top) 

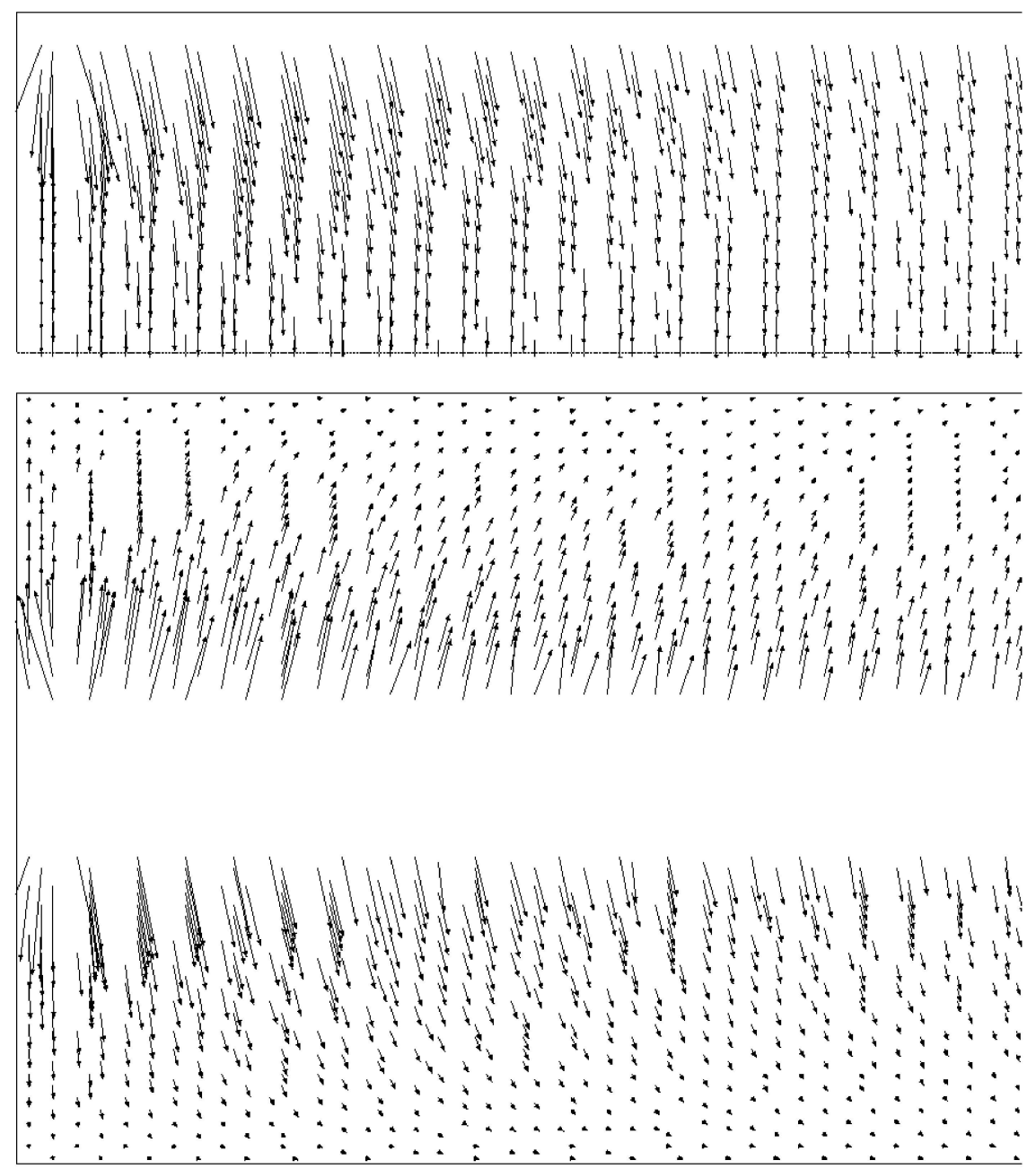

Fig. 2. Darcy velocity field in $X Z$ (top) and $X Y$ (bottom) cross-sections for the case of sand

subproblems was set to $10^{-12}$ instead of $10^{-6}$; second, the number of interior GMRES iterations for the Stokes problem in the Neumann-Dirichlet iterative coupling reduces from 110 to 21 , whereas the recovery of the iteration operator requires 160-180 GMRES iterations for each Stokes problem.

The iterative solution of the interface equation (28) is a very efficient procedure even in the case of realistic coefficients. We examined how the number of GMRES iterations depends on the critical parameters such as the mesh size $h$ and the conductivity $\mathbf{K}$.

In Table 2, we present the number of GMRES iterations, $\mathrm{N}_{\text {GMRES }}$, needed to reduce the initial residual of (28) (for zero initial guess) by a factor of $10^{-6}$, in the case of a material with $\mathbf{K}=\mathbf{I}$. The stopping criteria for the 

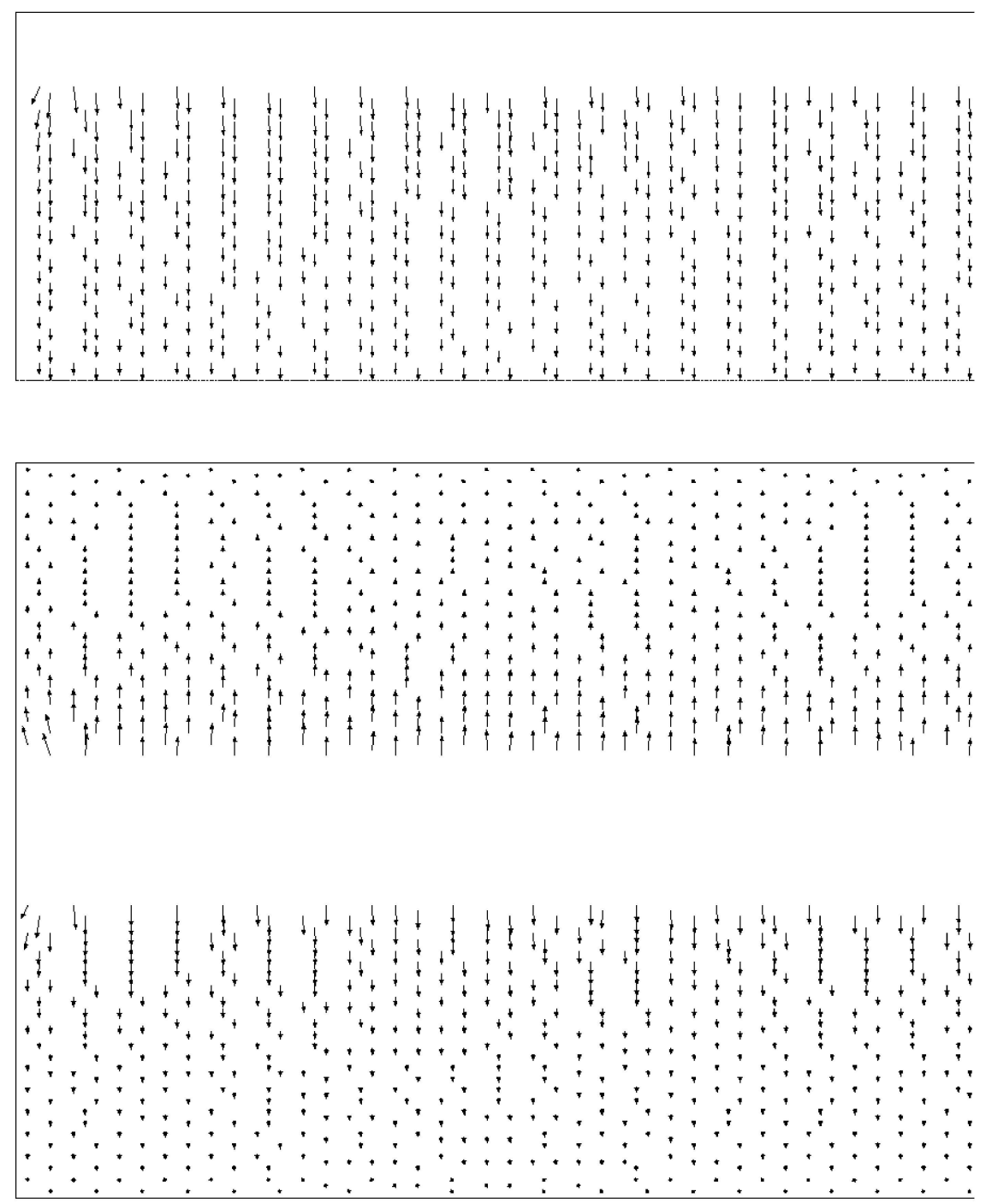

Fig. 3. Darcy velocity field in $X Z$ (top) and $X Y$ (bottom) cross-sections for the case of limestone

subdomain iterative solvers are residual reductions until $10^{-10}$ and $10^{-6}$ in $\Omega_{2}$ and $\Omega_{1}$, respectively. The number of degrees of freedom for $\Phi_{\Gamma}$ in (28) is denoted by \#DOF, the mean number of iterations applied to the Stokes and the Darcy subproblems are $\mathrm{N}_{\mathrm{St}}$ and $\mathrm{N}_{\mathrm{Dr}}$, respectively. The last column is the total computational time in seconds. The iteration counts for solving (28) and the Stokes subproblem are practically independent of mesh size, and $\mathrm{N}_{\text {Dr }}$ grows slowly with mesh refinement. The total computational time per coupling iteration is approximately proportional to the total number of 
Table 2. Iteration counts for the case $\mathbf{K}=\mathbf{I}$

\begin{tabular}{cccccc}
\hline Mesh & \#DOF & $\mathrm{N}_{\text {GMRES }}$ & $\mathrm{N}_{\mathrm{St}}$ & $\mathrm{N}_{\mathrm{Dr}}$ & $\mathrm{CPU}$ \\
\hline$k=4$ & 216 & 8 & 62 & 15 & 6 \\
$k=5$ & 429 & 8 & 53 & 20 & 74 \\
$k=6$ & 1625 & 9 & 53 & 23 & 1098 \\
\hline
\end{tabular}

Table 3. Iteration count $\mathrm{N}_{\mathrm{GMRES}}$ for different porous materials

\begin{tabular}{ccccccc}
\hline Mesh $\backslash \mathbf{K}=\mathbf{I} \times$ & 1 & $10^{-1}$ & $10^{-2}$ & $10^{-3}$ & $10^{-4}$ & $10^{-5}$ \\
\hline$k=4$ & 8 & 20 & 47 & 75 & 87 & 93 \\
$k=5$ & 8 & 20 & 42 & 64 & 72 & 73 \\
$k=6$ & 9 & 22 & 47 & 88 & 120 & 127 \\
\hline
\end{tabular}

Table 4. Spectrum of the interface operator (28) for $k=4$ and different porous materials

\begin{tabular}{ccccccc}
\hline Mesh $\backslash \mathbf{K}=\mathbf{I} \times$ & 1 & $10^{-1}$ & $10^{-2}$ & $10^{-3}$ & $10^{-4}$ & $10^{-5}$ \\
\hline$\lambda_{1}\left(\sigma_{1}\right)$ & $1(73)$ & $1(73)$ & $1(73)$ & $1(72)$ & $1(72)$ & $1(72)$ \\
$\lambda_{2}$ & 1.0026 & 1.026 & 1.26 & 3.62 & 27.2 & 263 \\
$\lambda_{\max }$ & 1.766 & 9.59 & 86.9 & 869 & 8589 & 85890 \\
\hline
\end{tabular}

unknowns, since each refinement multiplies the total number of unknowns by a factor of 8 .

Table 3 reflects the dependence on the conductivity coefficient $\mathbf{K}$. Two interesting effects can be observed in this table: saturation of $\mathrm{N}_{\mathrm{GMRES}}$ as $\mathbf{K} \rightarrow 0$ and non-monotone dependence of $\mathrm{N}_{\mathrm{GMRES}}$ on the mesh size for small conductivities. The first phenomenon is due to a very special structure of the spectrum of the interface operator $I+\left(S_{\mathcal{D}}^{h}\right)^{-1} M_{\lambda} P_{\mathbf{n}_{\Gamma}} \mathcal{C}^{-1} P_{\mathbf{n}_{\Gamma}}^{T} M_{\Phi_{\Gamma}}$ from (28) shown in Table 4. The spectrum is computed on the coarse grid, $k=4$. It consists of the marginal eigenvalue $\lambda_{1}=1$ with multiplicity $\sigma_{1}=72$ (73), and the remaining part belonging to the segment $\left[\lambda_{2}, \lambda_{\max }\right]$ whose both ends are scaled with $\|\mathbf{K}\|^{-1}$. For large conductivity coefficients, the marginal eigenvalue is not separated from the other eigenvalues, whereas $\lambda_{\max } \sim\|\mathbf{K}\|^{-1}$. As soon as the gap between $\lambda_{1}$ and $\lambda_{2}$ becomes large, the ratio $\lambda_{\max } / \lambda_{2}$ saturates. As a result, $\mathrm{N}_{\mathrm{GMRES}}$ saturates independently of the condition number $\lambda_{\max } / \lambda_{1}$ of the interface operator. This feature of GMRES iterations is similar to the property of the conjugate gradient method applied for spd matrices [1,7]. We cannot explain the non-monotone dependence of $\mathrm{N}_{\text {GMRES }}$ on mesh size for $\|\mathbf{K}\| \ll 1$ : the spectrum of the interface operator on medium mesh $(k=5)$ is similar to that presented in Table 4 except that the multiplicity of $\lambda_{1}$ is 88 . 


\section{Conclusions}

We considered several techniques for the iterative solution of coupled Darcy and Stokes flows. The study was motivated by the simulation of the interaction between channel flow and subsurface water flow in case of realistic coefficients and arbitrary interfaces between the different flow subdomains. The simplest iterative procedure, the Neumann-Dirichlet iteration, is not applicable due to its low convergence rate for the case of realistic coefficients. The recovery of the iteration operator was suggested as a possible remedy in the case of very slow convergence. Being a direct method, the recovery requires as many pairs of coupling iterations as the number of interface degrees of freedom. The recovery may be computationally expensive due to the higher precision required for the iterative solution of the subproblems. However, due to minimal data exchange, it provides the flexibility of using commercial software as subdomain solves.

An alternative approach is to derive an interface equation algebraically and apply the iterative solution based on Krylov subspace acceleration. The interface equation for the fluxes applies for plane interfaces between the flows. The interface equation for the normal stresses assuming continuous fluxes exhibits flexibility in the interface geometry and the benefits of the applicability of Krylov subspace iterative solvers. In spite of the huge stiffness of the discrete interface operator, the number of GMRES iterations only slightly depends on the values of the coefficients. This makes the iterative matching of the normal stresses (while keeping the fluxes continuous) very appealing in practical computations, although forming the interface equation hampers the use of commercial software as subdomain solves.

\section{References}

[1] Axelsson, O.: Iterative solution methods. Cambridge: Cambridge University Press 1994

[2] Beavers, G.S., Joseph, D.D.: Boundary conditions at a naturally permeable wall. J. Fluid Mech. 30, 197-207 (1967)

[3] Discacciati, M., Miglio, E., Quarteroni, A.: Mathematical and numerical models for coupling surface and groundwater flows. Appl. Numer. Math. 43, 57-74 (2002)

[4] Discacciati, M., Quarteroni, A.: Analysis of a domain decomposition method for the coupling of Stokes and Darcy equations. In: Brezzi, F. et al. (eds.): Numerical mathematics and advanced applications. ENUMATH 2001. Milan: Springer 2003, pp. 3-20

[5] Discacciati, M., Quarteroni, A.: Convergence analysis of a subdomain iterative method for the finite element approximation of the coupling of Stokes and Darcy equations. Comput. Vis. Sci. 6, 93-103 (2004)

[6] Discacciati, M.: Domain decomposition methods for the coupling of surface and groundwater flows. PhD Thesis, Ecole Polytechnique Federale de Lausanne 2004

[7] Graham, I., Hagger, M.J.: Unstructured additive Schwarz-conjugate gradient method for elliptic problems with highly discontinuous coefficients. SIAM J. Sci. Comput. 20, 2041-2066 (1999) 
[8] Jäger, W., Mikelić, A.: On the interface boundary condition of Beavers, Joseph and Saffman. SIAM J. Appl. Math. 60, 1111-1127 (2000)

[9] Layton, W., Schieweck, F., Yotov, I.: Coupling fluid flow with porous media flow. SIAM J. Numer. Anal. 40, 2195-2218 (2003)

[10] Miglio, E., Quarteroni, A., Saleri, F.: Coupling of free surface and groundwater flows. Comput. \& Fluids 32, 73-83 (2003)

[11] Payne, L.E., Straughan, B.: Analysis of the boundary condition at the interface between a viscous fluid and a porous medium and related modelling questions. J. Math. Pures Appl. (9) 77, 317-354 (1998)

[12] Porta, P.: Heterogeneous domain decomposition methods for coupled flow problems. PhD Thesis, University of Augsburg 2004

[13] Quarteroni, A., Valli, A.: Domain decomposition methods for partial differential equations. Oxford: Oxford University Press 1999

[14] Saffman, P.: On the boundary condition at the surface of a porous medium. Stud. Appl. Math. 50, 93-101 (1971)

[15] Salinger, A.G., Aris, R., Derby, J.J.: Finite element formulations for large-scale, coupled flows in adjacent porous and open fluid domains. Internat. J. Numer. Methods Fluids 18, 1185-1209 (1994)

[16] Stüben K.: Algebraic multigrid (AMG): experiences and comparisons. Appl. Math. Comput. 13, 419-451 (1983) 